\title{
Advances in optical metrology and instrumentation: introduction
}

\author{
Jonathan D. Ellis, ${ }^{1, *}$ (1) Han HaitJema, ${ }^{2}$ Xiangqian Jiang, ${ }^{3}$ KI-Nam Joo, ${ }^{4}$ and \\ RICHARD LEACH ${ }^{5}$ \\ ${ }^{1}$ Clerio Vision, Inc., 1892 S. Winton Road, Suite 140, Rochester, New York 14618, USA \\ ${ }^{2}$ Manufacturing Processes and Systems, Manufacturing Metrology, Department of Mechanical Engineering, KU Leuven, 3001 Leuven, Belgium \\ ${ }^{3}$ Department of Engineering and Technology, School of Computing and Engineering, University of Huddersfield, Huddersfield HD3 3PH, UK \\ ${ }^{4}$ Department of Photonic Engineering, Chosun University, 309 Pilmun-daero, Dong-gu, Gwangju, 61452, South Korea \\ ${ }^{5}$ Manufacturing Metrology Team, Faculty of Engineering, University of Nottingham, Nottingham NG8 1BB, UK \\ *Corresponding author: jellis@cleriovision.com
}

Received 14 August 2020; posted 14 August 2020 (Doc. ID 405559); published 31 August 2020

\begin{abstract}
Optical measurement and characterization are two of the pillars of metrology. The ability to measure precisely with high dynamic range and accuracy betters our understanding of nature and the universe. In this feature issue, we present a collection of articles that delves into the fundamental techniques used to advance the field. ( 2020 Optical Society of America
\end{abstract}

https://doi.org/10.1364/JOSAA.405559

Optical techniques allow the measurement of features on the atomic scale, for fields such as lithography, to the cosmic scale, where gravitational waves can be detected. While the uses of optical metrology are numerous, the fundamental techniques are relatively limited, and it is only when supported by specific instrumentation that truly novel science is enabled. Young [1] showed us the power of optical interference, and Michelson and Morley [2] demonstrated its application for dimensional metrology. But it was Maiman's invention of the laser [3] that unlocked the potential for coherent optical measurement over vast distances. This enabled advances in technology that led to the Laser Interferometer Gravitational-Wave Observatory (LIGO), which observed gravitational waves and confirmed a critical part of relativity theory [4]. This is but one example where fundamental principles for optical measurement and advances in instrumentation led to significant advances in our understanding of nature. The same can be said of advances in image sensors, detection, and signal processing.

The first paper in this feature issue is a tutorial [5] that provides a broad overview of currently implemented optical measurement techniques and expands on the rapidly evolving intersection between structured light and time of flight. Marrugo et al. detail some of the challenges associated with these techniques, providing potential avenues for future investigations.

Five articles presented in this issue cover optical measurement techniques and instrumentation for the small scale. de Groot and Colonna de Lega [6] propose a practical theoretical model for interference microscopes, including imaging properties when using partially coherence light. Their work elucidates that simplified models using coherent light and a linear, locally shift-invariant transfer function are valid within certain slope limitations and assuming optical aberrations are considered. Dickins et al. [7] seek to improve the development of additive manufacturing processes, such as metal powder bed fusion, by providing in-process measurement methods. In their work, the merits of single-view and multi-view fringe projection are compared and contrasted on additively manufactured components. Zorin et al. [8] propose an optical coherence tomography system that shifts the measurement spectrum to the mid-infrared range. Not only does this reduce scattering in three-dimensional imaging, but their implementation also enables morphological and chemical information to be obtained. Liu et al. [9] discuss the intersection between metrology and machine learning, which is a budding field of interest. Here, deep learning is used to mine information from light scattering patterns generated by surface defects. Fuerst et al. [10] demonstrate the use of the chromatic properties of light for simple point-to-point metrology. Their work shows the surface slope limitations of certain techniques.

Five further articles look to techniques on the meso- and macro-scale. Kim et al. [11] present work on absolute laser range finding using ultra-short pulses. Here, they demonstrate the power of frequency comb lasers, where both distance and refractive index information can be obtained from multiple objects simultaneously. Wisniewski et al. [12] present an integration of lasers and resonators for inertial sensing, demonstrating high accuracy frequency measurements and validating them through ancillary optical measurement techniques. Joo et al. [13] demonstrate displacement interferometer topologies with spatially separated beams and robust common paths to reduce 
influences from thermal effects, all the while not introducing periodic errors. Dubey and Kumar [14] present an intersection between classical measurement techniques and more modern techniques, where holographic shearing and Talbot shearing are explored. Furukawa et al. [15] explore interference and signal processing, demonstrating an extension of the measurement range beyond $\lambda / 2$ by analyzing the peak direction of the interference signal.

This feature issue demonstrates the innovative progresses in optical metrology and instrumentation. Looking to future challenges, with emerging technologies, e.g., nanophotonics, plasmonics, and metamaterials [16,17], data science, and machine learning with artificial intelligence, a new revolution in optical metrology will happen, after the previous revolutions brought about by the laser, advances in digital computing, and advances in detection technology. We hope this issue can inspire the next generation of researchers to take up these future and other currently unknown challenges.

\section{REFERENCES}

1. T. Young, "Il. The Bakerian Lecture. On the theory of light and colours," Philos. Trans. R. Soc. 92, 9212-9248 (1802).

2. A. A. Michelson and E. W. Morley, "On the relative motion of the Earth and the luminiferous ether," Am. J. Sci. s3-34, 333-345 (1887).

3. T. H. Maiman, "Ruby laser systems," U.S. Patent 3353115 (14 November 1967).

4. B. P. Abbott, LIGO Scientific Collaboration, and Virgo Collaboration, "Observation of gravitational waves from a binary black hole," Phys. Rev. Lett. 116, 061102 (2016).

5. A. G. Marrugo, F. Gao, and S. Zhang, "State-of-the-art active optical techniques for three-dimensional surface metrology: a review [Invited]," J. Opt. Soc. Am. A 37, B60-B77 (2020).
6. P. J. de Groot and X. Colonna de Lega, "Fourier optics modeling of interference microscopes," J. Opt. Soc. Am. A 37, B1-B10 (2020).

7. A. Dickins, T. Widjanarko, D. Sims-Waterhouse, A. Thompson, S. Lawes, N. Senin, and R. Leach, "Multi-view fringe projection system for surface topography measurement during metal powder bed fusion," J. Opt. Soc. Am. A 37, B93-B105 (2020).

8. I. Zorin, R. Su, B. Heise, B. Lendl, and M. Brandstetter, "Correlative infrared optical coherence tomography and hyperspectral chemical imaging," J. Opt. Soc. Am. A 37, B19-B26 (2020).

9. M. Liu, C. F. Cheung, N. Senin, S. Wang, R. Su, and R. Leach, "Onmachine surface defect detection using light scattering and deep learning," J. Opt. Soc. Am. A 37, B53-B59 (2020).

10. M. E. Fuerst, E. Csencsics, C. Haider, and G. Schitter, "Confocal chromatic sensor with an actively tilted lens for 3D measurement," J. Opt. Soc. Am. A 37, B46-B52 (2020).

11. W. Kim, J. Jang, S. Han, S. Kim, J. S. Oh, B. S. Kim, Y.-J. Kim, and S.-W. Kim, "Absolute laser ranging by time-of-flight measurement of ultrashort light pulses [Invited]," J. Opt. Soc. Am. A 37, B27-B35 (2020).

12. H. Wisniewski, L. Richardson, A. Hines, A. Laurain, and F. Guzmán, "Optomechanical lasers for inertial sensing," J. Opt. Soc. Am. A 37, B87-B92 (2020).

13. K.-N. Joo, E. Clark, Y. Zhang, J. D. Ellis, and F. Guzmán, "A compact high-precision periodic-error-free heterodyne interferometer," J. Opt. Soc. Am. A 37, B11-B18 (2020).

14. R. Dubey and R. Kumar, "Comparison of sensitivity to beam collimation of the holographic shearing interferometer with the wedge plate shearing interferometer and the Talbot shearing interferometer," J. Opt. Soc. Am. A 37, B36-B45 (2020).

15. O. Furukawa, S. Takemae, and Y. Tanaka, "Dynamic displacement measurement beyond half-wavelength in phase-modulated optical interferometer," J. Opt. Soc. Am. A 37, B78-B86 (2020).

16. "Top 10 Emerging Technologies 2019," https://www.weforum. org/reports/top-10-emerging-technologies-2019 (World Economic Forum, 2019).

17. G. Yuan and N. I. Zheludev, "Detecting nanometric displacements with optical ruler metrology," Science 364, 771-775 (2019). 